\title{
1. Introducing the collection
}

\author{
Silvia Pignata
}

This international collection addresses stress and well-being among workers across a variety of public sector roles and occupations. The public sector provides critical services to community members and there is a strong need for it to be effective, with human resource management being a significant contributor to its organizational success. In the private sector, there remains a perception that public sector employees have an easy life, and it is hoped that this collection will go some way to changing that perception. In reality, public sector organizations face ongoing and new challenges such as cost cutting, pressures to improve performance, changes in societal and workplace demographics, and increasing levels of stress among their employees. The current collection of empirical research, literature reviews, and case studies aim to draw greater attention to these and other challenges, and to provide practitioners with insights into how they can be managed. By doing so, the contributors hope to enhance the health and well-being of public sector employees, and the sector's performance and contribution to society.

\section{STRUCTURE OF THE BOOK}

The contributions in this book are from international experts in the field of work and organizational psychology, organizational studies, personnel psychology, management, clinical psychology, occupational health and safety management, health psychology, public health, clinical nursing practice, and policing and trauma. The diversity of countries represented (Australia, Canada, Italy, the Netherlands, United Kingdom (UK), United States (US)) reflects worldwide interest and efforts in this area.

The book is divided into four parts. In Part I, after this introduction, Chapter 2 provides an overview of well-being issues associated with the nature of work in the public sector and the need to embrace the characteristics of high-performing organizations. The role of UK trade unions in raising the nature of stress at work and how to respond positively within society is then examined in Chapter 3. Chapter 4 compares psychosocial factors and worker stress and well-being in the Australian private and public sectors and highlights the need for regular psychosocial risk assessments in both sectors.

Part II considers international research on the work, well-being, and treatment of workers in diverse public sector occupations (first responders, firefighters, nurses, prison officers, university faculty). The authors of Chapters 5-11, respectively, assert the need to foster resilience; address motivation issues; address mistreatment; manage emotions; consider the role of emotional intelligence in emotion manage- 
ment; identify and target well-being interventions; and use benchmarking approaches to enhance the health and quality of work life in academia. Current and emerging risk factors in the Italian public banking sector are then examined in Chapter 12.

Part III presents writings on successful public sector organizations, including case studies of efforts to bring about organizational change that also benefits individuals. The authors of Chapter 13 discuss the application of multidimensional psychological resources (i.e., psychological capital) in four contextual case studies - a fire department, the air force, a public school, and a university. Chapter 14 then considers the organizational benefits of developing and promoting proactive and adaptive individual performance such as efficacy training. In Chapters 15 and 16, researchers from Canada and the UK then detail the actions and commitment involved in building a health and safety culture and focusing on leadership and systems in health care settings.

Part IV emphasizes well-being, stress prevention, and the need for targeted and innovative initiatives to bring about effective change in workplaces. Successful practices are highlighted in case studies in order to inform policy on improving and sustaining worker health and well-being in both the public and private sectors (e.g., focusing on strategies to enhance positive relations, growth and purpose in order to develop nurse leaders in Chapter 17; detailing a new evidence-based well-being service for emergency service responders with interventions that include psychological screening, trauma risk management, and peer support programs in Chapter 18; initiating early peer counseling for first responders in Chapter 19; and reducing stress levels in the university context by developing and promoting targeted multilevel organizational interventions in Chapter 20).

\section{ACKNOWLEDGMENTS}

It is difficult to imagine a collection like this occurring without Ron Burke's immense contribution. I would also like to thank all the contributors who kindly agreed to share their work and their experiences in this handbook. 